\title{
Updates on Acute Myeloid Leukemia Management in older patients
}

\author{
Salman Otoukesh ${ }^{1 * *}$, Julio Alvarenga Thiebaud $^{2 *}$, Guido Marcucci ${ }^{1}$ and Monzr M Al Malki ${ }^{1}$ \\ ${ }^{1}$ Department of Hematology and Hematopoietic Cell Transplantation, Gehr center for Leukemia research, City of Hope National Medical Center, Duarte, CA, \\ USA \\ ${ }^{2}$ Bone Marrow Stem Cell Transplantation Fellow, Department of Hematology and Hematopoietic Cell Transplantation, Gehr center for Leukemia research, City \\ of Hope National Medical Center, Duarte, CA, USA

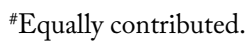

\begin{abstract}
Acute myeloid Leukemia (AML)'s incidence increases with age and more than $50 \%$ of patients are older than 60 years old. The treatment of AML in this age group population has been drastically changed since 2017 with all new innovative drugs approved by FDA highlighting the fact that "one size fit all" approach is not suitable to treat older patients with AML. However, the treatment of AML in elderly population yet to be defined as choosing the right treatment would depend on several other factors including performance status, comorbidities, along with diversities in leukemia subtype and characteristics (next gene sequencing, cytogenetics). Herein we have tried to focus on this specific age group and describe different treatment options (chemotherapy, immune checkpoint inhibitors, Bispecific T-cell engagers (BiTES), Chimeric Antigen Receptor T-cell Therapies (CAR-T), and Allogeneic Hematopoietic Stem Cell Transplantation) based on the available studies and ongoing clinical trials preliminary results.
\end{abstract}

\section{Introduction}

Acute Myeloid Leukemia (AML) is the most common type of acute leukemias in adults accounting for 80 percent of cases [1]. While AML is a relatively rare disease, it is more common in older adults, frequently diagnosed among people aged 65-74 with highest death in patients aged 75-84 [2]. Survival of patients with AML is heterogeneous and depends on several factors:(1) Patient related factors including performance status , Co-morbidities and organ function, (2) Disease related factors including type of AML (de novo Vs. Secondary), cytogenetics and molecular markers impacting outcomes $[1,3,4]$.

To better assess the prognosis of patients with AML, based on cytogenetics and molecular markers, the European Leukemia Net (ELN) proposed a system that identifies three prognostic risk groups that differ based on the rates of complete remission (CR), disease-free survival (DFS), and overall survival (OS) in the hopes of identifying potential new avenues of treatment for these heterogeneous disease entities [5].

Older Adults with AML (OA-AML) have worse survival than younger cohorts [6,7]. In a report from the Swedish Acute Leukemia Registry, early death rates in $\geq 70$ years old patients were five times more than those of $<50$ years old with 5 -year overall survival (OS) of $<10 \%$ among patients $\geq 70$ years old vs. $55 \%$ among those $<50$ years old; ECOG greater than 2 or having significant co-morbid conditions have been associated with worse outcome [8]. Adverse cytogenetics (i.e., loss of $5 \mathrm{q}, 7 \mathrm{q}$, and $17 \mathrm{p}$, monosomal karyotype and complex karyotypes) is another important factor which occurs more frequently in older patients leading to higher relapse rate and inferior outcomes compare with younger patients [9-11].
OA-AMLhave a higher frequency of mutations in genes known to be poor prognostic predictors [12,13]. For example, mutations of DNMT3A are reported in up to a third of OA-AML cases [14-16]. TP53 mutations along with complex karyotype (CK) leads to dismal outcomes; with 3 -year estimated EFS of $1 \%$ versus $13 \%$ (log-rank, P = $0.0007)$; RFS (relapse free survival) of $7 \%$ versus $30 \%(\mathrm{P}=0.01)$; and OS of $3 \%$ vs. $28 \%$ ( $\mathrm{P}<0.0001)$, after induction chemotherapy for CK/TP53 mutated and CK/TP53 wild-type patients respectively [17]. While hypomethylating agents and venetoclax seem to have improved results, TP53 mutated patients still suffer worse outcomes than WT [18]. Lastly, Other mutations such as ones seen in as IDH1/2, ASXL1, RUNX1, TET2, and BCOR also negatively affect outcomes in OA-AML[13,19].

\section{Treatment}

Traditionally, AML is treated with induction chemotherapy and depending on the risk category followed by consolidation with chemotherapy or Allogeneic hematopoietic cell transplantation (alloHCT). Unfortunately, data on the best choice of therapy in OAAML is sparse as several variables need to be considered before deciding which pathway to be used. Geriatric assessment (GA) should consist of a comprehensive evaluation of the patient's general functional status, falls, comorbidities, including psychological conditions, cognition,

${ }^{\star}$ Correspondence to: Salman Otoukesh, MD., Assistant Clinical Professor, Department of Hematology and HCT, City of Hope National Medical Center, 1500 East Duarte Rd, Duarte, CA 91010, USA, Tel: 626218 2405; Fax: 626389 3061; E-mail: sotoukesh@coh.org

Key words: older adults, AML, CAR-T, BiTES, AlloHCT

Received: May 05, 2021; Accepted: May 17, 2021; Published: May 20, 2021 
social support, and nutritional status. GA has been studied specifically in OA-AML, showing ability to better predict prognosis and potentially improving outcomes [20-22].

\section{Chemotherapy}

\section{Intensive chemotherapy (IC)}

The standard of care for the induction of AML remains cytarabine $200 \mathrm{mg} / \mathrm{m}^{2}$ continuous infusion $\mathrm{x} 7$ days with daunorubicin 60 or $90 \mathrm{mg} /$ $\mathrm{m} 2$ or Idarubicin $12 \mathrm{mg} / \mathrm{m} 2 \times 3$ days $(7+3)$ first described in the $1970 \mathrm{~s}$ $[23,24]$. Traditionally OA-AML treated with $7+3$ had worse outcomes. In a retrospective analysis of 968 adults with previously untreated AML, which investigated 3 clinical trials from Southwest Oncology Group (SWOG) [25-27], OA-AML treated with $7+3$ presented with poorer performance status, multidrug resistance (57\%) and unfavorable cytogenetics in $51 \%$ of patients older than 75 . Additionally, OA-AML had a CR rate of $33 \%$, median OS of 3.5 months, and DFS of 8.9 months, while young patients had a CR rate of $64 \%$, median OS 18.8 months, and DFS of 21.6 months regardless the presence of favorable cytogenetics [9]. In the AML-14 trial, a total of 1273 patients were recruited addressed different dose regimens for Daunorubicin and Cytarabine in addition to the multidrug resistance modulator PSC-833 in a $1: 1: 1$ randomization in patients predominantly aged over 60 years with AML and High-Risk MDS. The study showed an overall response rate (ORR) of $62 \%$ (complete remission (CR) $54 \%$, complete remission without platelet/neutrophil recovery (CRi) $8 \%$ ), with 5-year survival rate of $12 \%$ without improving outcomes in older patients [28]. In 2009, 813 patients older than 60 years of age were randomized to escalated-dose of daunorubicin $\left(90 \mathrm{mg} / \mathrm{m}^{2}\right)$ Vs. conventional dose $\left(45 \mathrm{mg} / \mathrm{m}^{2}\right)$, leading to higher rates of complete remission ( $73 \%$ vs. $51 \%)$, event-free survival ( $29 \%$ vs. $14 \%$ ), and overall survival ( $38 \%$ vs. $23 \%$ ) when compared to the conventional dose regimen, without additional toxic effects [29].

Fortunately, over the past decades, a wave of novel arsenal of various therapies has drastically altered the treatment landscape of AML, especially in the elderly. One such therapy is Vyxeos (CPX351) which is a liposomal formulation of daunorubicin and cytarabine that releases the drugs in a fixed 5:1 molar ratio and was examined in a randomized phase II study on patients age 60 to 75 years with newly diagnosed AML, first-line CPX-351 showed a trend to higher response rates $(66.7 \%$ vs. $51.2 \%, P=.07)$, improved 60 -day mortality $(4.7 \%$ vs. $14.6 \%)$ with $\mathrm{OS}(12.1$ vs. 6.1 months; $\mathrm{HR}=0.46, \mathrm{P}=.01)$ in sAML subset when compared to $7+3$ [30]. Based on these results, an openlabel, randomized, phase III trial enrolled 309 elderly patients with newly diagnosed high-risk or sAML received one to two induction cycles of CPX-351 $7+3$ followed by consolidation therapy. This trial showed that CPX-351 vs. $7+3$ significantly improved median OS (9.56 v 5.95 months; $\mathrm{P}=0.003$ ) and ORR (Overall Response Rate) ( $47.7 \%$ v $33.3 \%$; two-sided $\mathrm{P}=.016$ ), with improved outcomes observed across age-groups and AML subtypes [31]. These studies ultimately led to the approval by the FDA in 2017 of CPX-351 for the treatment of adults with newly diagnosed AML with myelodysplasia-related changes (AML-MRC) or therapy-related acute myeloid leukemia ( $t$-AML). The 5 -year follow-up results from the phase 3 study demonstrated persistent improved OS with CPX-351 vs. $7+3$ chemotherapy in the overall study population, but the survival rates were still dismal (21\% vs. $9 \%$ and $18 \%$ vs. $8 \%)$ at 3 and 5 years, respectively in both groups respectively [32]. Aspacytarabine (BST-236) is a novel cytarabine prodrug designed to reduced toxicity by decreasing the peak exposure of free cytarabine in unfit patients. The phase $1 / 2$ and phase 2 studies involving $42 \mathrm{AML}$ patients with a median age of 73 years. Forty-three percent of patients with adverse cytogenetics attained CR, with median OS for sAML group of 6.8 months, not reached for the de novo AML patients with good safety profile with a 30 -day mortality rate of $7 \%$. [33].

\section{Hypomethylating agents}

Hypomethylating agents have also emerged as therapeutic alternatives in OA-AML management. Decitabine (DEC) and Azacytidine (AZA) have been evaluated in this patient population. The DACO-016 study reported the efficacy and safety of decitabine vs. treatment choice (TC; supportive care or cytarabine) in 485 older patients with newly diagnosed AML with the median age of 73 years old, The primary analysis with 396 deaths $(81.6 \%)$ showed a nonsignificant increase in median OS with decitabine (7.7 months; $95 \%$ CI, 6.2 to 9.2) versus TC (5.0 months; $95 \% \mathrm{CI}, 4.3$ to $6.3 ; P=.108$; hazard ratio [HR], $0.85 ; 95 \% \mathrm{CI}, 0.69$ to 1.04$)$. The CR / CRi rate was $17.8 \%$ with decitabine versus $7.8 \%$ with TC (odds ratio, $2.5 ; 95 \% \mathrm{CI}, 1.4$ to $4.8 ; P=.001$ ), and the adverse events were similar between the two groups [34]. These findings led to the approval in Europe of Decitabine for patients aged $\geq 65$ years with de novo/secondary AML who are ineligible for intensive therapy. The AZA-AML-001 trial was a multicenter, randomized, open-label, phase 3 study that evaluated efficacy and safety of AZA vs. conventional care regimens (CCRs; standard induction chemotherapy, L-DAC, or supportive care only) in 488 elderly patients with newly diagnosed AML and $>30 \%$ bone marrow blasts. The median OS with AZA was 10.4 months (95\% confidence interval [CI], 8.0-12.7 months) vs 6.5 months in the CCR group (95\% CI, 5.0-8.6 months) with a hazard ratio of 0.85 (95\% CI, 0.69-1.03; stratified log-rank $\mathrm{P}=.1009$ ). Additionally, the one-year survival estimate of $50.7 \%$ in AZA compare to 33.8 in CCR (difference, $16.9 \%$; $95 \%$ CI, $1.5 \%, 32.2 \%$ ) put AZA as a valid treatment option for this difficult-to-treat AML population [35]. Moreover, a recent report from the Pethema registry found no significant differences in ORR, CR/CRi, or OS between DEC and AZA [36]. HMA have been used in combination with chemotherapy also. One such combination is low dose cytarabine (LDAC) alternating with clofarabine and decitabine. In 2015, Kadia, et al. reported a phase 2 study using this regimen in 180 elderly patients with an ORR of $68 \%$ and a CR of $60 \%$. The median OS was 11.1 months for all patients and 18.5 months for those achieving CR/CRi with four and eightweek mortality rates of $3 \%$ and $7 \%$, respectively [37]. This same group in 2018 reported their experience with LDAC in combination with Cladribine alternating with decitabine on 118 patients with $58 \% \mathrm{CR}$ rate, median OS of 13.8 months and DFS of 10.8 months. Similar to clofarabine study, the four and eight-week mortality rates were $1 \%$ and $7 \%$, respectively [38]. Targeted agents have also been successfully used in combinations with these agents, as explained below.

\section{BCL-2 Inhibitors}

Venetoclax (VEN) is now considered as category 1 recommendation in combination with HMA in ELP who cannot tolerate high-dose chemotherapy regimens [39]. VEN is a BCL-2 inhibitor on the leukemic cells which are dependent on MCL-1 for their survival [40-42]. The effectiveness of VEN monotherapy was reported in a phase 2 single-arm study of 32 patients with high-risk relapsed/refractory AML (RR AML) or in those who were unfit to receive intensive chemotherapy. The ORR was $19 \%$, with an additional $19 \%$ of patients demonstrated antileukemic activity not meeting IWG criteria [43]. Based on these results and the synergistic activity observed in preclinical data, combinations therapies were studied. DiNardo, et al. reported the combination of VEN and HMA, a phase $1 \mathrm{~b}$ study of 57 elderly patients who were ineligible for standard induction, demonstrated ORR of $75 \%$ (95\% CI 62.2-85.9) 
with $61 \%$ of patients achieving CR/CRi regardless of HMA type. The median duration of response (mDOR) was 8.4 months, with a median OS of 12.3 months [44]. These results were confirmed by the VIALE-A phase 3 trial which 431 OA-AML (median age 76 years, range 49-91) were randomly assigned to AZA plus VENVE (AZA/VEN) or placebo. After a median follow-up of 20.5 months, the CR/Cri and median OS were $66.4 \%$ vs. $28.3 \%(\mathrm{P}<0.001)$ and 14.7 vs. 9.6 months (HR for death, 0.66; $95 \% \mathrm{CI}, 0.52$ to $0.85 ; \mathrm{P}<0.001)$ in the AZA/VEN and control groups, respectively, additionally mortality rate at 30 day remained similar in two groups (7\% Vs. 6\%) [45]. Compared to intensive chemotherapy (IC), decitabine combined with VEN (DEC/VEN) has also shown better outcomes; Maiti, et al. reported a propensity scorematched analysis stratified by risk of treatment-related mortality of 85 older adults with median age of 72 years (range 63-89). After a median follow-up of 12.4 months in DV and 81.2 months in the IC cohort, they found a significantly higher CR/CRi of $81 \%$ vs. $52 \%(\mathrm{P}<.001)$, with a longer OS of 12.4 vs. 4.5 months, respectively ( $\mathrm{HR}=0.48,95 \% \mathrm{CI}$ $0.29-0.79, \mathrm{P}<.01)$ [46].

VEN also has been combined with other agents to try improving outcomes. VEN plus low dose cytarabine (VEV-LDAC) was studied in a phase 3 randomized placebo-controlled trial of 211 patients (2:1) with median age of 76 years (range 36-93) and after a median follow up of 18 months, the median OS was 8.4 months (95\% CI, 5.9-10.1) for V-LDAC vs 4.1 months (95\% CI, 3.1-8.1) for those receiving placebo with an overall response rate of $48 \%$ versus $13 \%(\mathrm{p}<0.001)$, and a CR rate of $27 \%$ versus $7 \%(\mathrm{p}<0.001)$ respectively [47]. Other chemotherapy combination regimens have also been successful, but they have not been studied in the elderly $[48,49]$. Isocitrate dehydrogenase (IDH) $1 / 2$ mutations have been shown to cause increased leukemia cell sensitivity to VEN likely related to inhibition of cytochrome $\mathrm{c}$ oxidase in the electron transport chain, thus lowering the threshold for triggering VEN induced apoptosis [50]. This synergism was confirmed clinically on the phase II monotherapy (VEN) study, which found increased activity in patients with AML treated with VEN who harbored IDH1/2 mutations, with a third of them achieving CR/CRi [43]. Similar findings were reported in those patients treated with V-LDAC [51]. Lachowiez, et al. in 2020 reported the results of a phase $1 \mathrm{~b} / 2$ study of Ivosidenib (IVOSI) with VEN +/- AZA in 19 patients with IDH1-mutated hematologic malignancies; seventeen patients had AML, with a median age of 68 years. In 18 evaluable patients, the composite complete remission (CRc: $\mathrm{CR}+\mathrm{CRi}+\mathrm{CRh}$ ) rates was $78 \%$ (treatment-naive: $100 \%, \mathrm{R} / \mathrm{R}: 75 \%$ ) with a median time to best response of 2 months. After a median follow-up of 3.5 months, in treatment naïve patients, median OS was not reached (9.7 months in R/R patients) [52]. FLT3 inhibitors are another group of agents under investigation in addition to VEN for the management of AML. In a recently reported phase $1 \mathrm{~B}$ study, Daver, et al. investigated the safety and efficacy of VEN combined with Gilteritinib for 39 patients with RR FLT3 mutated AML. While not specifically looking into just an elderly population, the average age of the population was 63 years. it was found a modified composite CR (CR + CRp + CRi) of $83 \%$ but due to the short follow-up of a large number of recently enrolled patients, the interpretation is limited [53].

\section{Isocitrate Dehydrogenase 1 and 2 Inhibitors}

Recurrent mutations in IDH1 and IDH2 produced 2-hydroxyglutarate (2HG) from $\alpha$-KG, preventing the histone demethylation leading to a block in differentiation and leukemogenesis $[54,55]$. IVOSI and Enasidenib (ENASI) are oral IDH1 and IDH2 inhibitors which have been approved as monotherapies for RR AML; A phase 1 , multicenter, open-label study treated 60 patients with
IVOSI plus induction chemotherapy and 93 patients with ENASI plus induction chemotherapy with the median age of 62.5 and 63 years in the IVOSI and ENASI cohorts, respectively. The CR/CRi/CRp at the end of induction was $72 \%$, and $63 \%$ with IVOSI and ENASI, respectively. After a follow-up of 9.3 and 14.5 months, median OS was not reached and 14.5 with 12 -months survival of $78 \%$ and $76 \%$ in both IVOSI and INASI treated cohorts; respectively [56]. The HOVON150 AML trial (NCT03839771) is a phase 3, multicenter, double-blind, randomized, placebo-controlled study of IVOSI or ENASI in combination with induction therapy and consolidation therapy followed by maintenance therapy currently recruiting patients with newly diagnosed AML or MDS with excess blasts-2 who harbor an IDH1 or IDH2 mutation. IDH1 and IDH2 inhibitors have been also combined with HMA. In a phase $1 \mathrm{~b} / 2$ study reported by DiNardo, et al. where 17 patients were treated with AZA and either IVOSI $(n=11)$ or INASI $(n=6)$; ORR reported $72 \%$ (8 patients) and $66 \%$ (4 cases) with CR rate of $36 \%$ (4 cases) and 33\% ( 2 cases), respectively [57]. Similar results was reported by this group in a different phase $1 \mathrm{~b}$ (NCT02677922) combining IVOSI plus AZA n 23 patients with median age of 76 years (range 61-88); median follow up of 16 months with ORR of $78.3 \%$ and CR 60.9\% [58].

\section{Drug-Antibody Conjugates}

Gemtuzumab Ozogamicin (GO) is a humanized recombinant antiCD33 antibody (hP67.6) conjugated to calicheamicin, an antitumor antibiotic [59]. In AML, Larson, et al. reported the results of three multicenter, open-label, phase 2 studies that evaluated the efficacy and safety of GO monotherapy $\left(9 \mathrm{mg} / \mathrm{m}^{2}\right.$ in 2 doses separated by 14 days) in 277 AML patients, including 157 of which were age 60 years or older. The ORR was $26 \%$ without a significant difference between young and elderly patients with the median OS of 4.9 months. In those patients who entered remission after receiving $>2$ doses of GO, the OS was 12.6 months vs. 4.2 months in those who still had evidence of disease [60]. Based on these results, GO was initially approved by the FDA to manage AML patients who were not candidates for IC. Unfortunately, the SWOC S0106 trial results ultimately showed increased in treatment-related mortality related to GO leading to the withdrawal of the medication [61]. Then in the ALFA-0701, a phase 3 , open-label, multicenter study, of 280 previously untreated de novo AML with CD33 positivity randomized to standard chemotherapy with or without GO at $3 \mathrm{mg} / \mathrm{m}^{2}$ on days $1,4,7$, the authors found a CR rate of $81 \%$ in the experimental group with improved OS $53.2 \%$ vs. $41.9 \%$ and relapsed free survival (RFS) of $50.3 \%$ vs. $22.7 \%$ in the GO group without increasing risk of death due to toxicity, 17 patients in GO group whom achieved CR/CRp could receive SCT and venoocclusive disease occurred in 3 of them [62]. In the randomized phase 3 EORTC-GIMEMA AML-19 Trial, GO as single agent was compared to BSC in OA-AML with median age of 77 years (range 62-88) unsuitable for IC; A total of 247 patients were treated with an ORR of $27 \%$ with 1 year OS of $24.3 \%$ vs. $9.7 \%$ in the GO and BSC groups, respectively. no excess in mortality related to toxicities was found in either arms [63]. Moreover, a meta-analysis of five randomized controlled trials of 3325 patients found that GO reduced risk or relapse and improved OS. The authors also found that doses of $3 \mathrm{mg} / \mathrm{m} 2$ were just as effective as $6 \mathrm{mg} /$ $\mathrm{m} 2$ but associated with fewer early deaths [64]. These results led to the re-approval of the medication by the FDA.

\section{FLT3 Inhibitors}

Fms-like tyrosine kinase 3 (FLT3) is a protein that belongs to the class III family of receptor tyrosine kinase (RTK) that plays a key role in controlling the survival, proliferation, and differentiation of 
hematopoietic cells. FLT3 mutations occur in about a third of elderly with AML, resulting in a constitutively activated FLT3 kinase, leading to proliferation and survival of AML $[65,66]$. For this reason, FLT3 inhibitors (FLT3i) have an important role in the treatment of AML, as evidenced by the improved OS in the pivotal phase 3 RATIFY trial, which compared the addition of Midostaurin (MIDO) to $7+3$ induction vs. chemotherapy alone. Unfortunately, no patients $>60$ years of age were enrolled in this trial however these data can be considered for elderly fit patients as well [67]. However in a phase II study of adding MIDO to IC followed by consolidation with stem cell transplantation or HiDAC and receiving MIDO as maintenance in a mixed age groups (18-70 years old, with $30 \%$ more than 60 years old) demonstrated CR/ CRi rate of 75.8 Vs.77.9\% in age $<60$ years old and elderly, respectively, $72.4 \%$ of patients could go for transplantation followed by MIDO maintenance in 75 patients while the rest $(27 \%)$ received HiDAC consolidation followed by MIDO maintenance. The 2-year EFS and OS were $39 \%$ and $34 \%$ Vs. $53 \%$ and $46 \%$ in patients with age $<60$ years old and older respectively [68]. Gilteritinib, another FLT3i, showed antileukemic activity in combination with AZA in patients ineligible for intensive induction in the first in human phase $1 / 2$ study (CR 67\%) [69]. In the randomized phase 3 ADMIRAL trial Giltritinib was used in R/R AML setting with the median age of patients was 62 years demonstrating significant longer survival and CR/CRi compare to salvage chemotherapy group (9.3 vs. 5.6 months; $\mathrm{p}<0.001$ and $34 \%$ vs. $15.3 \%$, respectively [70]. Qusartinib is another selective and highly potent oral FLT3i type II which was investigated in QuANTRUM-R phase 3 randomized trial, although the median age was less than 60 years, it showed longer overall survival (HR 0.76, p 0.02) and median overall survival compare to preselected chemotherapies (LDAC, FLAGIDA, MEC) group ( 6.2 vs. 4.7 months, respectively) [71]. Sorafenib is another member of this family and was used as a first line treatment (in unfit elderly with untreated FLT3-ITD mutated to receive IC) in combination with AZA ( $\mathrm{n}=27$, age 61-86 years old) demonstrating ORR $78 \%$ with CR/CRi rate of $70 \%$ and median OS og 8.3 for all group [72].

\section{TP53 Targeted Therapies}

The p53 is a tumor suppressor gene that triggers cell cycle arrest, and or apoptosis on cellular stress. TP53 mutation is found in about $10 \%$ of OA-AMLand in up to $70 \%$ of cases with complex karyotype (CK) [18 65]. For many years, no option was available to target this mutation. In 2002, the first compound that could restore sequence-specific DNAbinding, wild-type conformation, and transcriptional transactivation to mutant p53 was reported [73]. Ten years later, Lehmann, et al. described the first-in-human study of Eprenetapopt (APR-246), a methylated form of the original compound, in hematologic malignancies [74]. Then, in a phase $1 \mathrm{~b} / 2$ open-label, multicenter, dose-escalation, and dose-expansion study of treatment naïve MDS/AML patients with a median age of 74 years, the combination of AZA and Eprenetapopt found an ORR of $64 \%$ and CR of $36 \%$ in AML patients and a median OS of 10.8 months [75]. Magrolimab (MAGRO) is another medication that has shown activity in TP53 mutated OA-AML. It is a first-in-class antibody targeting CD47, a macrophage immune checkpoint, and a "don't eat me" signal on cancers. A phase $1 \mathrm{~b}$ study combining MAGRO/ AZA in AML/MDS patients with a median age of 73 years found an ORR of $69 \%$ in AML patients with 50\% CR with median survival of 12.9 vs. 18.9 months in TP53- mutant and wild-type, respectively; however subgroup analysis also revealed that $88 \%$ of TP53 mutated patients achieved an objective response. [76,77].

\section{Hedgehog pathway inhibitors}

Glasdegib is an antagonist of the Hedgehog pathway through binding to Smoothened that can decrease dormant AML leukemic stem cells burden [78]. Based on this information, Cortes, et al. reported a randomized trial comparing LDAC with and without glasdegib in 132 patients with newly diagnosed AML/MDS; more than half of the population was over 75 years of age. After a median follow-up of 21.7 months, median OS in AML group was 8.3 vs 4.4 months in glasdegib vs. LDAC alone arm. Additionally, the ORR was $26.9 \%$ vs. $5.3 \%$ in the glasdegib and LDAC vs. LDAC alone [78]. Because LDAC is not commonly used in the United States, Shallis, et al. announced a multicenter, randomized phase 2 study evaluating the efficacy of glasdegib combined with two different doses of decitabine in newly diagnosed poor-risk elderly AML patients who either refuse or are ineligible for intensive therapy (NCT04051996). This trial is ongoing.

\section{Immune Checkpoint Inhibitors}

Checkpoint inhibitors have become one of the cornerstones in the management of oncologic diseases. Physiologically, they regulate self-tolerance and protect tissue against the potential damage that the immune system can cause by adjusting the signals that mediate T-cell immune response [79]. In AML, there seems to be immune suppression at the level of the bone marrow mediated by overexpression of PD-1 compared to healthy individuals [80]. As with other agents, immune checkpoint inhibitors have been used in the management of relapse/ refractory (RR) AML, but to date, no data have been published, specifically in the elderly as front line. Pembrolizumab in combination with Decitabine currently is under recruiting patients with newly diagnosed/RR AML or myelodysplastic syndrome (MDS) in a phase IB trial (NCT03969446). A phase II study of High dose cytarabine (HiDAC) followed pembrolizumab RR AML enrolled $41 \%$ of the population with an age of 60 years or older. The ORR and composite CR (CR+CRi) rates were $46 \%$ [29\%,63\%] and 38\% [22\%,55\%], respectively, meeting the primary endpoint of the study. After a median followup of 7.8 months, the median OS was 8.9 months (NCT02768792). In another phase $1 \mathrm{~B} / 2$ study of nivolumab in combination with azacitidine for RR AML; This single-arm trial enrolled patients with a median age of 70 years to receive treatment with both medications. The ORR was $33 \%$, with a CR rate of $22 \%$ and a median OS of 6.3 months [81]. In the frontline setting, the combination of pembrolizumab with chemotherapy was evaluated in a phase 2 trial investigating the use of $7+3$ and pembrolizumab induction for newly diagnosed AML or MDS. The median age was 54 years, with $20 \%$ of patients being over 60 years of age. After a median follow-up of 17.25 months, $55 \%$ of patients were alive (with a median of 18.5 months) with ORR (CR+CRi+CRp) of $78 \%$ (34/44) [82]. Unfortunately, the outcomes of the elderly patients were not described separately. Therefore, further data is needed to define the role of these medications in the management of AML in OA-AML.

\section{Bispecific T-cell engagers (BiTE)}

Bispecific T-cell engagers are antibody constructs that link T cells to tumor antigens, leading to activation of cytotoxic response [83]. Several BiTEs are currently being evaluated for AML; Flotetzumab is a CD123/ CD3 BiTE studied in a phase $1 / 2$ study of 122 patients (median age of $>60$ years) with primary induction failure (PIF), early relapse (ER), or RR AML. The ORR for RR and PIF/ER was $24 \%$ and $30 \%$, respectively. The study also reported 6- and 12-month survival rates of $42 \%(0.237$, $0.596)$ and $20 \%(0.025,0.377)$ for PIF/ER $(n=30)$, respectively. Most patients experienced some degree of cytokine release syndrome (CRS), 
but most of them $(81 \%)$ had mild to moderate symptoms. Neurologic involvement was infrequent, seen in only $10 \%$ of cases [84]. AMG 330 is a BiTE that binds to CD33 and CD3. In a phase 1, open-label doseescalation study, 55 patients with a median age of 58 years, including individuals up to 80 years of age, reported an ORR of $19 \%$. No survival data have been reported, and this study is still ongoing [85]. Other BiTEs, such as AMG673, AMG 427, AMV564, and XmAb14045, are currently under study. While some responses have also been seen, they are modest at best in early phase trials [86-88].

\section{Chimeric Antigen Receptor T-Cell Therapy (CAR-T)}

Currently, ACT is administered as genetically engineered Chimeric Antigen Receptor (CAR) T cells [89]. This treatment modality has successfully treated refractory hematologic malignancies (ALL, Lymphoma's, multiple myeloma), as evidenced by high remission rates in this high-risk patient population [90-93]. AML, as previously mentioned, is a heterogeneous disease, and therefore finding an optimal target has been challenging. To date, different CAR-T's have been used in the setting of in-vivo AML with promising findings [94-99] and currently several AML CAR-T trials targeting different antigens like CD33 (NCT03971799), CD38 (NCT04351022), CD123 (NCT03190278), and NKG2D (NCT02203825), are underway mainly in the relapse-refractory setting, though not specifically for the elderly population.

\section{Allogeneic Hematopoietic Stem Cell Transplantation}

Despite all the advances AML still is associated with poor outcomes in the elderly however, in a large multicenter retrospective study (alliance A151509, SWOG, ECOG-ACRIN, and CIBMTR) comparing outcomes of patients consolidated with allo-HSCT vs. more chemotherapy indicating allo-HSCT had more Treatment-Related Mortality (TRM) in the first 9 months (p 0.0009) but with significantly reduced relapse rate beyond 9 months $(\mathrm{p}<0.0001)$ and superior long term OS at 5 years (29\% vs. $13.8 \%$ ) [100,101]. The role of myeloablative conditioning regimen (MAC) in elderly is not robust mainly because of more co-morbidities in patients and TRM in MAC compare to Reduced intensity regimen (RIC). The CALBG 100103 phase 2 study prospectively assessed the efficacy of RIC in patients (age of 60 to 74 years) with AML in CR1. The study authors found a 2-year OS of $42 \%$ with disease free survival (DFS) of 50\%, NRM 15\%, and Cumulative Incidence of Relapse (CIR) of 44\% [102]. A meta-analysis of 13 studies reported an OS of $38 \%$ at 3 years with a corresponding relapse free survival (RFS) of $35 \%$ and NRM of $40 \%$ [103]. More recently, Del Galy, et al. reported their real-world experience in OA-AML treated with IC or HMA followed by reduced-intensity conditioning (RIC) alloHSCT; Of the entire cohort, the transplantation rate was $35 \%$ in those achieving CR with a median age of 68 years. With a median follow-up of 44 months, the estimated OS was 50 months in patients who underwent transplant vs. 20.6 months in no-transplant group. Additionally TRM was $<5 \%$, with 2 years relapse rate of $45 \%$ contributing using RIC instead of MAC in a group of elderly patients with median HCT-CI of 3 [104]. While myeloablative conditioning (MAC) regimens have also been used with a reported 3 years OS, NRM, and relapse rate of $34 \%$, $43 \%$, and $24 \%$, respectively [105]. For these reasons, RIC allo-HSCT is considered the most effective therapy to obtain durable remissions in OA-AML. This benefit is believed to be related to its graft vs. leukemia (GVL) effect in addition to better supportive care [106]. Unfortunately, about $<10 \%$ of OA-AML get transplanted despite of the clear benefit in survival.

\section{Conclusions}

In conclusion, OA-AML is a population at increased risk for poor outcomes related not only to underline hematological malignancies but also to underlying physiologic deterioration related to age. In the management of this patient population multiple factors should be taken into consideration (performance status, disease status, gene mutation profile, etc.) before choosing the treatment. While new therapies have proven to be effective in the management of OA-AML, long term follow up is needed to determine the impact on outcomes. Lastly, if the individual can tolerate, RIC allo HSCT should be offered to these patients as is the single most important treatment that has been repeatedly shown to improve long-term disease-free survival (DFS) in this population.

\section{Conflict of Interest disclosure}

GM: speakers' bureau for AbbVie and Novartis, advisory board with Janssen, MAL: advisory board with Gilead. The rest of the authors with no conflict of interest.

\section{Funding}

This study was supported by intramural funds from City of Hope National Medical Center.

\section{References}

1. https://seer.cancer.gov/statfacts/html/amyl.html

2. Medeiros BC, Satram-Hoang S, Hurst D, Hoang KQ, Momin F, et al. (2015) Big data analysis of treatment patterns and outcomes among elderly acute myeloid leukemia patients in the United States. Ann Hematol 94: 1127-38. [Crossref]

3. Siegel RL, Miller KD, Jemal (2020) Cancer statistics. CA: A Cancer Journal for Clinicians. 70: 7-30.

4. Cancer Facts \& Figures 2019 (2019) American Cancer Society: Atlanta.

5. Papaemmanuil E, Gerstung M, Bullinger L, Gaidzik VI, Paschka P, et al. (2016) Genomic Classification and Prognosis in Acute Myeloid Leukemia. N Engl J Med 374: 2209-2221. [Crossref]

6. Juliusson G (2011) Older Patients With Acute Myeloid Leukemia Benefit From Intensive Chemotherapy: An Update From the Swedish Acute Leukemia Registry. Clin Lymphoma Myeloma Leuk 11: S54-S59. [Crossref]

7. Williams JP, Handler HL (2000) Antibody-targeted chemotherapy for the treatment of relapsed acute myeloid leukemia. Am J Manag Care 6: S975-S985. [Crossref]

8. Juliusson G, Antunovic P, Derolf A, Lehmann S, Möllgård L, et al. (2009) Age and acute myeloid leukemia: real world data on decision to treat and outcomes from the Swedish Acute Leukemia Registry. Blood 113: 4179-4187. [Crossref]

9. Appelbaum FR, Gundacker H, Head DR, Slovak ML, Willman CL, et al. (2006) Age and acute myeloid leukemia. Blood 107: 3481-3485. [Crossref]

10. Büchner T, Berdel WE, Haferlach C, Haferlach T, Schnittger S, et al (2009) AgeRelated Risk Profile and Chemotherapy Dose Response in Acute Myeloid Leukemia: A Study by the German Acute Myeloid Leukemia Cooperative Group. J Clin Oncol 27: 61-69. [Crossref]

11. Lazarevic V, Hörstedt AS, Johansson B, Antunovic P, Billström R, et al. (2014) Incidence and prognostic significance of karyotypic subgroups in older patients with acute myeloid leukemia: the Swedish population-based experience. Blood Cancer J 4: e188-e188. [Crossref]

12. Silva P, Neumann M, Schroeder P, Vosberg S, Schlee C, et al. (2017) Acute myeloid leukemia in the elderly is characterized by a distinct genetic and epigenetic landscape. Leukemia 31: 1640-1644. [Crossref]

13. Victoria VP, Rothenberg-Thurley M, Sauerland MC, Herold T, Janke H, et al. (2018) Genetics of acute myeloid leukemia in the elderly: mutation spectrum and clinical impact in intensively treated patients aged 75 years or older. Haematologica 103: 18531861. [Crossref]

14. Ley TJ, Ding L, Walter MJ, McLellan MD, Lamprecht T, et al. (2010) DNMT3A Mutations in Acute Myeloid Leukemia. N Engl J Med 363: 2424-2433. [Crossref] 
15. Marcucci G, Metzeler KH, Schwind S, Becker H, Maharry K, et al. (2012) Age-related prognostic impact of different types of DNMT3A mutations in adults with primary cytogenetically normal acute myeloid leukemia. J Clin Oncol 30: 742-750. [Crossref]

16. Yan XJ, Xu J, Gu ZH, Pan CM, Lu G, et al. (2011) Exome sequencing identifies somatic mutations of DNA methyltransferase gene DNMT3A in acute monocytic leukemia. Nat Genet 43: 309-315. [Crossref]

17. Rücker FG, Schlenk RF, Bullinger L, Kayser S, Teleanu V, et al. (2012) TP53 alterations in acute myeloid leukemia with complex karyotype correlate with specific copy number alterations, monosomal karyotype, and dismal outcome. Blood 119: 2114-2121. [Crossref]

18. DiNardo CD, Pratz K, Pullarkat V, Jonas BA, Arellano M, et al. (2019) Venetoclax combined with decitabine or azacitidine in treatment-naive, elderly patients with acute myeloid leukemia. Blood 133: 7-17. [Crossref]

19. Tsai CH, Hou HA, Tang JL, Liu CY, Lin CC, et al. (2016) Genetic alterations and their clinical implications in older patients with acute myeloid leukemia. Leukemia 30: 1485-1492. [Crossref]

20. KlepinA HD (2014) Feasibility of geriatric assessment for older adults with acute myeloid leukemia (AML) receiving intensive chemotherapy on a cooperative group trial: CALGB 361006 (Alliance). Journal of Clinical Oncology 32: 7102-7102.

21. Klepin HD, Geiger AM, Tooze JA, Kritchevsky SB, Williamson JD, et al. (2013) Geriatric assessment predicts survival for older adults receiving induction chemotherapy for acute myelogenous leukemia. Blood 121: 4287-4294. [Crossref]

22. Sherman AE, Motyckova G, Fega KR, Deangelo DJ, Abel GA, et al. (2013) Geriatric assessment in older patients with acute myeloid leukemia: a retrospective study of associated treatment and outcomes. Leuk Res 37: 998-1003. [Crossref]

23. NCCN (2021) NCCN Clinical Practice Guidelines in Oncology (NCCN Guidelines ${ }^{\circledR}$ ). Acute Myeloid Leukemia.

24. Yates JW, Wallace Jr HJ, Ellison RR, Holland JF (1973) Cytosine arabinoside (NSC63878) and daunorubicin (NSC-83142) therapy in acute nonlymphocytic leukemia. Cancer Chemother Rep 57: 485-488. [Crossref]

25. Godwin JE, Kopecky KJ, Head DR, Willman CL, Leith CP, et al. (1998) A doubleblind placebo-controlled trial of granulocyte colony-stimulating factor in elderly patients with previously untreated acute myeloid leukemia: a Southwest oncology group study (9031). Blood 91: 3607-3615. [Crossref]

26. Anderson JE, Kopecky KJ, Willman CL, Head D, O’Donnell MR, et al. (2002) Outcome after induction chemotherapy for older patients with acute myeloid leukemia is not improved with mitoxantrone and etoposide compared to cytarabine and daunorubicin: a Southwest Oncology Group study. Blood 100: 3869-3876. [Crossref]

27. Petersdorf S (1998) A phase II study of standard dose daunomycin and cytosine arabinoside (Ara-C) with high-dose Ara-C induction therapy followed by sequential high-dose Ara-C consolidation for adults with previously untreated acute myelogenous leukemia: a Southwest Oncology Group study (SWOG 9500). In Proc Annu Meet Am Soc Clin Oncol.

28. Burnett AK, Milligan D, Goldstone A, Prentice A, McMullin MF, et al. (2009) The impact of dose escalation and resistance modulation in older patients with acute myeloid leukaemia and high risk myelodysplastic syndrome: the results of the LRF AML14 trial. Br J Haematol 145: 318-332. [Crossref]

29. Löwenberg B, Ossenkoppele GJ, Putten WV, Schouten HC, Graux C, et al. (2009) High-Dose Daunorubicin in Older Patients with Acute Myeloid Leukemia. $N$ Engl J Med 361: 1235-1248. [Crossref]

30. Lancet JE, Cortes JE, Hogge DE, Tallman MS, Kovacsovics TJ, et al. (2014) Phase 2 trial of CPX-351, a fixed 5:1 molar ratio of cytarabine/daunorubicin, vs cytarabine/ daunorubicin in older adults with untreated AML. Blood 123: 3239-3246. [Crossref]

31. Lancet JE, Uy GL, Cortes JE, Newell LF, Lin TL, et al. (2018) CPX-351 (cytarabine and daunorubicin) Liposome for Injection Versus Conventional Cytarabine Plus Daunorubicin in Older Patients With Newly Diagnosed Secondary Acute Myeloid Leukemia. J Clin Oncol 36: 2684-2692. [Crossref]

32. Lancet JE (2020) Five-year Final Results of a Phase 3 Study of CPX-351 Versus 7+ 3 in Older Adults With Newly Diagnosed High-risk/Secondary AML. changes (AMLMRC). $4: 5$

33. Altman JK (2020) Durable Remissions and Increased Overall Survival in AML Patients Deemed Unfit for Standard Intensive Chemotherapy Achieved with High-Dose BST236 (Aspacytarabine) Induction and Consolidation. Blood 136: 9-10.

34. Kantarjian HM, Thomas XG, Dmoszynska A, Wierzbowska A, Mazur G, et al. Multicenter, randomized, open-label, phase III trial of decitabine versus patient choice, with physician advice, of either supportive care or low-dose cytarabine for the treatment of older patients with newly diagnosed acute myeloid leukemia. J Clin Oncol 30: 2670-2677. [Crossref]
35. Dombret H, et al. (2015) International phase 3 study of azacitidine vs conventional care regimens in older patients with newly diagnosed AML with $>30 \%$ blasts. Blood 126 : 291-299. [Crossref]

36. Labrador J (2020) Azacitidine Vs. Decitabine in Unfit Newly Diagnosed Acute Myeloid Leukemia Patients: Results from the Pethema Registry. Blood 136: 25-27.

37. Kadia TM, Fader S, Ravandi F, Jabbour E, Garcia-Manero G, et al. (2015) Final results of a phase 2 trial of clofarabine and low-dose cytarabine alternating with decitabine in older patients with newly diagnosed acute myeloid leukemia. Cancer 121: 2375-2382. [Crossref]

38. Kadia TM, Cortes J, Ravandi F, Jabbour E, Konopleva M, et al. (2018) Cladribine and low-dose cytarabine alternating with decitabine as front-line therapy for elderly patients with acute myeloid leukaemia: a phase 2 single-arm trial. Lancet Haematol 5 : e411-e421. [Crossref]

39. NCCN (2021) Guidelines Version 3.2021 Acute Myeloid Leukemia (Age >18 years).

40. Pan R, Hogdal LJ, Benito JM, Bucci D, Han L, et al. (2014) Selective BCL-2 Inhibition by ABT-199 Causes On-Target Cell Death in Acute Myeloid Leukemia. Cancer Discov 4: 362-375. [Crossref]

41. Vo TT, Ryan J, Carrasco R, Neuberg D, Rossi DJ, et al. (2012) Relative Mitochondria Priming of Myeloblasts and Normal HSCs Determines Chemotherapeutic Success in AML. Cell 151: 344-355. [Crossref]

42. Opferman JT, Iwasaki H, Ong CC, Suh H, Mizuno S, et al. (2005) Obligate Role of Anti-Apoptotic MCL-1 in the Survival of Hematopoietic Stem Cells. Science 307: 1101-1104. [Crossref]

43. Konopleva M, Pollyea DA, Potluri J, Chyla B, Hogdal L, et al. Efficacy and Biological Correlates of Response in a Phase II Study of Venetoclax Monotherapy in Patients with Acute Myelogenous Leukemia. Cancer Discov 6: 1106-1117. [Crossref]

44. DiNardo CD, Pratz KW, Letai A, Jonas BA, Wei AH, et al. (2018) Safety and preliminary efficacy of venetoclax with decitabine or azacitidine in elderly patient with previously untreated acute myeloid leukaemia: a non-randomised, open-label, phase 1b study. Lancet Oncol 19: 216-228. [Crossref]

45. DiNardo CD (2020) Azacitidine and Venetoclax in Previously Untreated Acute Myeloid Leukemia. N Engl J Med 383: 617-629.

46. Maiti A, Qiao w, Sasaki k, Ravandi F, Kadia TM, et al. (2021) Venetoclax with decitabine vs intensive chemotherapy in acute myeloid leukemia: A propensity score matched analysis stratified by risk of treatment-related mortality. Am J Hematol 96 282-291. [Crossref]

47. Wei AH, Montesinos P, vanov V, DiNardo CD, Novak J, et al. (2020) Venetoclax plus LDAC for newly diagnosed AML ineligible for intensive chemotherapy: a phase 3 randomized placebo-controlled trial. Blood 135: 2137-2145. [Crossref]

48. Lachowiez C, Kadia TM, Daver N, Loghavi S, Wang SA, et al. (2020) Interim Analysis of the Phase $1 \mathrm{~b} / 2$ Study of the BCL-2 Inhibitor Venetoclax in Combination with Standard Intensive AML Induction/Consolidation Therapy with FLAG-IDA in Patients with Newly Diagnosed or Relapsed/Refractory AML.

49. Reville PK, Borthakur G, Yilmaz M, Montalban-Bravo G, DiNardo CD, et al. (2020) Cladribine, Idarubicin, Cytarabine (ara-C), and Venetoclax in Treating Patients with Acute Myeloid Leukemia and High-Risk Myelodysplastic Syndrome.

50. Chan SM, Thomas D, Corces-Zimmerman MR, Xavy S, Rastogi S, et al. (2015) Isocitrate dehydrogenase 1 and 2 mutations induce BCL-2 dependence in acute myeloid leukemia. Nat Med 21: 178-184. [Crossref]

51. Wei AH, Strickland Jr SA, Hou J, Fiedler W, Lin TL, et al. (2019) Venetoclax Combined With Low-Dose Cytarabine for Previously Untreated Patients With Acute Myeloid Leukemia: Results From a Phase Ib/II Study. J Clin Oncol 37: 1277-1284. [Crossref]

52. Lachowiez CA (2020) Phase Ib/II study of the IDH1-mutant inhibitor ivosidenib with the BCL2 inhibitor venetoclax +/- azacitidine in IDH1-mutated hematologic malignancies. J Clin Oncol 38: 7500-7500.

53. Daver N, Maly J, Levis M, Ritchie E, Litzow M, et al. (2020) Efficacy and Safety of Venetoclax in Combination with Gilteritinib for Relapsed/Refractory FLT3-Mutated Acute Myeloid Leukemia in the Expansion Cohort of a Phase 1b Study.

54. Lu, C, Ward PS, Kapoor GS, Rohle D, Turcan S, et al. (2012) IDH mutation impairs histone demethylation and results in a block to cell differentiation. Nature 483: 474478. [Crossref]

55. Losman JA, Looper RE, Koivunen P, Lee S, Schneider RK, et al. (2013) (R)-2 hydroxyglutarate is sufficient to promote leukemogenesis and its effects are reversible. Science 339: 1621-1625. [Crossref] 
56. Stein EM, DiNardo CD, Fathi AT, Mims AS, Pratz KW, et al. (2021) Ivosidenib or enasidenib combined with intensive chemotherapy in patients with newly diagnosed AML: a phase 1 study. Blood 137: 1792-1803. [Crossref]

57. Dinardo CD (2018) Mutant IDH (mIDH) inhibitors, ivosidenib or enasidenib, with azacitidine (AZA) in patients with acute myeloid leukemia (AML). J Clin Oncol 36: 7042-7042.

58. DiNardo CD, Stein AS, Stein EM, Fathi AT, Frankfurt O, et al. (2021) Mutant Isocitrate Dehydrogenase 1 Inhibitor Ivosidenib in Combination With Azacitidine for Newly Diagnosed Acute Myeloid Leukemia. J Clin Oncol 39: 57-65. [Crossref]

59. Hamann PR, Hinman LM, Hollander I, Beyer CF, Lindh D, et al. (2002) Gemtuzumab Ozogamicin, A Potent and Selective Anti-CD33 Antibody-Calicheamicin Conjugate for Treatment of Acute Myeloid Leukemia. Bioconjug Chem 13: 47-58. [Crossref]

60. Larson RA, Sievers EL, Stadtmauer EA, Löwenberg B, Estey EH, et al. (2005) Final report of the efficacy and safety of gemtuzumab ozogamicin (Mylotarg) in patients with CD33-positive acute myeloid leukemia in first recurrence. Cancer 104: 1442-1452. [Crossref]

61. Petersdorf S (2009) Preliminary Results of Southwest Oncology Group Study S0106: An International Intergroup Phase 3 Randomized Trial Comparing the Addition of Gemtuzumab Ozogamicin to Standard Induction Therapy Versus Standard Induction Therapy Followed by a Second Randomization to Post-Consolidation Gemtuzumab Ozogamicin Versus No Additional Therapy for Previously Untreated Acute Myeloid Leukemia. Blood 114: 790-790.

62. Castaigne S, Pautas C, Terré C, Raffoux E, Bordessoule D, et al. (2012) Effect of gemtuzumab ozogamicin on survival of adult patients with de-novo acute myeloid leukaemia (ALFA-0701): a randomised, open-label, phase 3 study. Lancet 379: 15081516. [Crossref]

63. Amadori S, Suciu S, Selleslag D, Aversa F, Gaidano G, et al. (2016) Gemtuzumab Ozogamicin Versus Best Supportive Care in Older Patients With Newly Diagnosed Acute Myeloid Leukemia Unsuitable for Intensive Chemotherapy: Results of the Randomized Phase III EORTC-GIMEMA AML-19 Trial. J Clin Oncol 34: 972-979. [Crossref]

64. Hills RK, Castaigne S, Appelbaum FR, Delaunay J, Petersdorf S, et al. (2014) Addition of gemtuzumab ozogamicin to induction chemotherapy in adult patients with acute myeloid leukaemia: a meta-analysis of individual patient data from randomised controlled trials. Lancet Oncol 15: 986-996. [Crossref]

65. Stirewalt DL, Kopecky KJ, Meshinchi S, Appelbaum FR, Slovak ML, et al. (2001) FLT3, RAS, and TP53 mutations in elderly patients with acute myeloid leukemia. Blood 97: 3589-3595. [Crossref]

66. Grafone T, Palmisano M, Nicci C, Storti S et al. (2012) An overview on the role of FLT3-tyrosine kinase receptor in acute myeloid leukemia: biology and treatment. Oncol Rev 6: e8-e8. [Crossref]

67. Stone RM (2017) Midostaurin plus Chemotherapy for Acute Myeloid Leukemia with a FLT3 Mutation. New England Journal of Medicine 377: 454-464.

68. Schlenk RF, Weber D, Fiedler W, Salih HR, Wulf G, et al. (2019) Midostaurin added to chemotherapy and continued single-agent maintenance therapy in acute myeloid leukemia with FLT3-ITD. Blood 133: 840-851. [Crossref]

69. Perl AE, Altman JK, Cortes J, Smith C, Litzow M, et al. (2017) Selective inhibition of FLT3 by gilteritinib in relapsed or refractory acute myeloid leukaemia: a multicentre, first-in-human, open-label, phase 1-2 study. Lancet Oncol 18: 1061-1075. [Crossref]

70. Perl AE, Martinelli G, Cortes JE, Neubauer A, Berman E, et al. (2019) Gilteritinib or chemotherapy for relapsed or refractory FLT3-mutated AML. $N$ Engl J Med 381: 1728-1740. [Crossref]

71. Cortes JE, Khaled S, Martinelli G, Perl AE, Ganguly S, et al. (2019) Quizartinib versus salvage chemotherapy in relapsed or refractory FLT3-ITD acute myeloid leukaemia (QuANTUM-R): a multicentre, randomised, controlled, open-label, phase 3 trial. Lancet Oncol 20: 984-997. [Crossref]

72. Ohanian M, Garcia-Manero G, Levis M, Jabbour E, Daver N, et al. (2018) Sorafenib combined with 5-azacytidine in older patients with untreated FLT3-ITD mutated acute myeloid leukemia. Am J Hematol 93: 1136-1141. [Crossref]

73. Bykov VJN, Issaeva N, Shilov A, Hultcrantz M, Pugacheva E, et al. (2002) Restoration of the tumor suppressor function to mutant $\mathrm{p} 53$ by a low-molecular-weight compound. Nat Med 8: 282-288. [Crossref]

74. Lehmann S, Bykov VJN, Ali D, Andrén O, Cherif H, et al. (2012) Targeting p53 in Vivo: A First-in-Human Study With p53-Targeting Compound APR-246 in Refractory Hematologic Malignancies and Prostate Cancer. J Clin Oncol 30: 3633-3639. [Crossref]
75. https://library.ehaweb.org/eha/2020/eha25th/295001/thomas.cluzeau.apr-246. combined.with.azacitidine.in.tp53.mutated.html?f=listing\%3D0\%2Abrowseby\%3D8 \%2Asortby\%3D1\%2Asearch\%3Dapr-246+combined\%2Bazacitidine

76. Sallman DA (2019) The First-in-Class Anti-CD47 Antibody Magrolimab (5F9) in Combination with Azacitidine Is Effective in MDS and AML Patients: Ongoing Phase 1b Results. Blood 134: 569-569.

77. https://library.ehaweb.org/eha/2020/eha25th/294964/naval.daver.the.first-inclass.anti-cd47.antibody.magrolimab.combined.with.html? $=$ menu $\% 3 \mathrm{D} 6 \% 2 \mathrm{Ab}$ rowseby $\% 3 \mathrm{D} 8 \% 2 \mathrm{Asortby} \% 3 \mathrm{D} 2 \% 2 \mathrm{Amedia} \% 3 \mathrm{D} 3 \% 2 \mathrm{Ace}$ id $\% 3 \mathrm{D} 1766 \% 2 \mathrm{Aot}$ id\%3D23221\%2Amarker\%3D756

78. Sadarangani A, Pineda G, Lennon KM, Chun H, Shih A, et al. (2015) GLI2 inhibition abrogates human leukemia stem cell dormancy. J Transl Med 13: 98. [Crossref]

79. Chen L, Flies DB (2013) Molecular mechanisms of T cell co-stimulation and coinhibition. Nat Rev Immunol 13: 227-242. [Crossref]

80. Daver N (2016) Defining the Immune Checkpoint Landscape in Patients (pts) with Acute Myeloid Leukemia (AML). Blood 128: 2900-2900.

81. Daver N, Garcia-Manero G, Basu S, Boddu PC, Alfayez M, et al. (2019) Efficacy, safety, and biomarkers of response to azacitidine and nivolumab in relapsed/refractory acute myeloid leukemia: a nonrandomized, open-label, phase II study. Cancer Discov 9: 370-383. [Crossref]

82. Ravandi F, Assi R, Daver N, Benton CB, Kadia T, et al. (2019) Idarubicin, cytarabine, and nivolumab in patients with newly diagnosed acute myeloid leukaemia or highrisk myelodysplastic syndrome: a single-arm, phase 2 study. Lancet Haematol 6: e480-e488. [Crossref]

83. Baeuerle PA, Reinhardt C (2009) Bispecific T-Cell Engaging Antibodies for Cancer Therapy. Cancer Res 69: 4941-4944. [Crossref]

84. Uy GL, Aldoss I, Foster MC, Sayre PH, Wieduwilt MJ, et al. (2021) Flotetuzumab as salvage immunotherapy for refractory acute myeloid leukemia. Blood 137: 751-762. [Crossref]

85. Ravandi F (2020) Updated results from phase I dose-escalation study of AMG 330, a bispecific T-cell engager molecule, in patients with relapsed/refractory acute myeloid leukemia (R/R AML). Journal of Clinical Oncology 38: 7508-7508.

86. Subklewe M (2019) Preliminary results from a phase 1 first-in-human study of AMG 673, a novel half-life extended (HLE) anti-CD33/CD3 BiTE®(bispecific T-cell engager) in patients with relapsed/refractory (R/R) acute myeloid leukemia (AML) American Society of Hematology Washington, DC.

87. Westervelt P (2019) Phase 1 first-in-human trial of AMV564, a bivalent bispecific (2: 2) CD33/CD3 T-cell engager, in patients with relapsed/refractory acute myeloid leukemia (AML). Blood 134: 834.

88. Ravandi F (2018) Complete responses in relapsed/refractory acute myeloid leukemia (AML) patients on a weekly dosing schedule of XmAb14045, a CD123 x CD3 T cellengaging bispecific antibody: initial results of a phase 1 study. American Society of Hematology Washington, DC.

89. Morgan RA, Dudley ME, Rosenberg SA (2010) Adoptive cell therapy: genetic modification to redirect effector cell specificity. Cancer $J$ 16: 336-341. [Crossref]

90. Schuster SJ, Svoboda J, Chong EA, Nasta SD, Mato AR, et al. (2017) Chimeric Antigen Receptor T Cells in Refractory B-Cell Lymphomas. N Engl J Med 377: 2545 2554. [Crossref]

91. Neelapu SS (2017) Axicabtagene Ciloleucel CAR T-Cell Therapy in Refractory Large B-Cell Lymphoma. $N$ Engl J Med 377: 2531-2544.

92. Raje N, Berdeja J, Lin Y, Siegel D, Jagannath S, et al. (2019) Anti-BCMA CAR T-Cell Therapy bb2121 in Relapsed or Refractory Multiple Myeloma. $N$ Engl J Med 380 1726-1737. [Crossref]

93. Maude SL (2018) Tisagenlecleucel in Children and Young Adults with B-Cell Lymphoblastic Leukemia. N Engl J Med 378: 439-448.

94. Itoh-Nakadai A (2020) CXCR4-expressing anti-CD25 CAR T-cells effectively eliminate human AML cells in vivo. in Blood. 2020. Amer Soc Hematology WASHINGTON, DC, USA.

95. Driouk L, Gicobi JK, Kamihara Y, Rutherford K, Dranoff G, et al. Chimeric antigen receptor T cells targeting NKG2D-ligands show robust efficacy against acute myeloid leukemia and T-cell acute lymphoblastic leukemia. Front Immunol 11: 580328. [Crossref] 
96. Wermke M, Kraus S, Ehninger A, Bargou RC, Goebeler M, et al. (2021) Proof-ofconcept for Rapidly Switchable Universal CAR-T Platform with UniCAR-T-CD123 in Relapsed/Refractory AML. Blood 23: 2020009759. [Crossref]

97. Leivas A (2019) NKG2D CAR-Expressing Lymphocytes Target Acute Myeloid Leukemia Cells. American Society of Hematology Washington, DC.

98. Hasegawa A, Saito S, Narimatsu S, Nakano S, Nagai M, et al. (2020) Mutated GMCSF-Based CAR T-Cells Targeting CD116/CD131 Complexes Exhibit Enhanced Anti-Tumor Effects Against Acute Myeloid Leukemia. Clin Transl Immunology 10 e1282. [Crossref]

99. Srinivasan S (2020) Investigation of ALLO-316: A fratricide-resistant allogeneic CAR $\mathrm{T}$ targeting CD70 as a potential therapy for the treatment of AML. Blood.

100. Juliusson G (2019) Improved survival of men 50 to 75 years old with acute myeloid leukemia over a 20-year period. Blood 34: 1558-1561. [Crossref]

101. Ustun C, Le-Rademacher J, Wang HL, Othus M, Sun Z, et al. (2019) Allogeneic hematopoietic cell transplantation compared to chemotherapy consolidation in older acute myeloid leukemia (AML) patients 60-75 years in first complete remission (CR1): An alliance (A151509), SWOG, ECOG-ACRIN, and CIBMTR study. Leukemia 33: 2599-2609. [Crossref]
102. Devine SM, Owzar K, Blum W, Mulkey F, Stone RM, et al. (2015) Phase II Study of Allogeneic Transplantation for Older Patients With Acute Myeloid Leukemia in First Complete Remission Using a Reduced-Intensity Conditioning Regimen: Results From Cancer and Leukemia Group B 100103 (Alliance for Clinical Trials in Oncology)/Blood and Marrow Transplant Clinical Trial Network 0502. J Clin Oncol 33: 4167-4175. [Crossref]

103. Rashidi A, Ebadi M, Colditz GA, DiPersio JF, et al. (2016) Outcomes of Allogeneic Stem Cell Transplantation in Elderly Patients with Acute Myeloid Leukemia: A Systematic Review and Meta-analysis. Biol Blood Marrow Transplant 22: 651-657. [Crossref]

104. Del Galy AS, Marouf A, Raffoux E, Robin M, Michonneau D, et al. (2021) Allogeneic hematopoietic stem cell transplantation in elderly patients with acute myeloid leukemia or myelodysplastic syndromes: myth and reality. Leukemia 35 : 225-228. [Crossref]

105. Wallen H, Gooley TA, Deeg HJ, Pagel JM, Press OW, et al. (2005) Ablative Allogeneic Hematopoietic Cell Transplantation in Adults 60 Years of Age and Older. J Clin Oncol 23: 3439-3446. [Crossref]

106. Storb R (2007) Can reduced-intensity allogeneic transplantation cure older adult with AML? Best Pract Res Clin Haematol 20: 85-90. [Crossref]

Copyright: $\odot 2021$ Otoukesh S. This is an open-access article distributed under the terms of the Creative Commons Attribution License, which permits unrestricted use, distribution, and reproduction in any medium, provided the original author and source are credited. 